\title{
Digital Housing
}

\author{
Charles Vincent ${ }^{1}$, Luiz Alberto Fresl Backheuser ${ }^{2}$, \\ Eduardo Campolongo ${ }^{3}$ \\ ${ }^{1,2}$ Mackenzie Presbyterian University ${ }^{3}$ Mackenzie Presbyterian university \\ ${ }^{1,2}\left\{\right.$ charles.vincent|luiz.backheuser\}@mackenzie.br ${ }^{3}$ eduardocampolongo@hotmail. \\ com
}

This paper exposes some of the experiments and results attained in a broader research project carried along in our University. The research is focused on the development of a wiki-like house building system, derived from preceding cases.It goes space from layout generation through full-scale prototyping of a new system and it's intended to further reduce construction costs while retaining some flexibility in space layout and dealing with consequent variations in construction components.

Keywords: Digital Housing, Fabrication, Prototyping, Wiki-House

\section{INTRODUCTION}

Digital Housing research is part of a broader effort to acquire and develop digital technologies in a developing country context. The main goal is to acquire, analyze, redesign and program a complete housing construction system, derived from but not constrained to the WikiHouse concept. The project is specifically tailored to Brazil's economic and technological reality.In the Wikihouse community, a philosophical - ideological principle seems to underlay the majority of the initiatives. Wikihouse is presented as a system that decentralizes and distributes knowledge in the form of a pre-programmed construction package. Wikihouse.cc website states as their mission: "1. To put the design solutions for building lowcost, low-energy, high-performance homes into the hands of every citizen and business on earth.2. To use digitization to make it easier for existing industries to design, invest-in, manufacture and assemble better, more sustainable, more affordable homes for more people.3. To grow a new, distributed housing industry, comprising many citizens, communities and small businesses developing homes and neighborhoods for themselves, reducing our dependence on top-down, debt-heavy mass housing systems." (Wikihouse.cc, 2018)At first, those principles would easily meet the requisites defining Industry 4.0 , that is, Interconnection, Information Transparency, Technical assistance, Decentralized decisions.It so happens that in developing countries those requisites are far away from reality, at least in cities and regions removed from the biggest economic centers.Most experiments carried in research centers and universities in Latin America rely on digital infrastructure present in those centers, and so is our research. This research thus encompasses the study and production of a complete CNC machined construction system ready for delivery and assembly in construction sites. Main topics of research are: Wiki-like systems comparisonComponentized construction logicsParametric and constraint-based designSystem redesign for greater economy in developing countries. This article focuses specifically on the Digital Housing parametric and constraint-based aspects of the research. 


\section{METHODS}

The complete research comprises the following aspects: 1.Wiki-like systems comparison: Our research has focused on the understanding of three different wiki-like systems.2. Componentized construction logic. The study has focused on understanding the principles of modular coordination in wood framing construction and its application in wiki-like systems. 3. Parametric and constraint-based design. Departing from previous studies in parametric and constraint-based design, the study seeks to deliver a programmed system for the generation of wikilike design variations.4. System redesign for greater economy in developing countries. Parallel to that, the research intends to attain greater economy in material use while granting reasonable energetic and structural efficiencies.

\section{PRECEDENTS}

Literature with a specific focus in wikihouse and similar fabrication systems is scarce and usually very shallow. Lawrence Sass seems to have the most consistent bibliographic production on the subject, mainly related to his research on the Instant House. Sass's papers don't expose much of the system's construction details. On the other hand, its Shape Grammar approach to systematize construction logic is unique, deriving from other authors' Shape Grammar studies.

Wikihouse foundation is somehow more centered in the social aspects and its website has a clear emphasis on the description of the wiki construction system and strategies related to it. In Wikihouse construction logic is embedded - although not in its full extents - into the WREM system, currently developed in Rhino - Grasshopper. A rather important experiment was also carried out in Rio de Janeiro, at Universidade Federal do Rio de Janeiro, led by Clarisse Rohde in 2015.

FACIT (London - Bell Travers Willson, 2007) adopts the same principles Wikihouse and the Instant House: precut CNC machining of panels from a digital project where fittings are precut. The main difference in FACIT's system components is its pre- assembled "cassettes" which are interconnected insitu. It might be added that FACIT was developed into a full commercial fabrication system and is now a fledgling architectural business. The FACIT prototype was not compared to the other three systems in this research.

ClipHut, an initiative at Ostwestfalen-Lippe University of Applied Sciences, adopts the same CNC pre-machined components logics, but instead of plywood, it adopts OSB panels for the structure cassettes. Those systems are being studied in a rather systematic way. Each one has been redrawn in CAD - 2d and 3d and then reprogrammed in Grasshopper. From the Grasshopper parametric definition, scale models in $1 / 3$ and $1 / 6$ scales are generated, machined and mounted.

Each studied system is then examined against a series of checkpoints, the principal ones being the joint system logic, degree of complexity in machining patterns, cassettes' structural soundness, ease of assembly.In the ideological realm, the study is influenced by an urge to make the system more participatory, in the sense conveyed in "[...] that design in collaboration is not only associated with the Seventies, but that this is a modern, even postmodern notion which provides us with the basis for both social and scientific development in design theory." (Bax, 1995)

The final design for the research group Housing Project comprises the redesign of the whole system from the ground up. Main goals are: Substantial reduction in material consumption while retaining structural soundness. Substantial reduction in joint machining and thus a reduction in machining time, Coordination between the componentized system and more traditional dry construction materials and methods.

\section{EXPERIMENTS}

In order to coherently coordinate fabricated components into a constructive grid, software needs some control over plain boards dimensions and a sound structural spacing between structural cassettes. A completely constrained system would, in fact, per- 
Figure 1

Grasshopper screen capture: script to generate Plans and Massings from Python code

Figure 2

Grasshopper Screen capture: Plan for a 4 occupants dwelling. mit an extremely great number of plan variables. On the other hand, a less constrained system can accommodate for a broader range of finish panel dimensions, flexibility necessary for an uneven market such as Brazil. This has led the team to adjust grasshopper programming to simultaneously allow for closed systems - modularity and joints logic - and adaptability to variations in panel dimensions. As for modularity control, the research established an array of possible interconnections among partitions related to a constructive grid, in what Shawcross describes as a "Severely Constrained Design" (Shawcross, 2013)

The team has worked with Wikihouse Wren 4.1 and 4.2 as the base system for testing and results comparison, since it's the best documented and more widespread, making access to other experimentations more secure. However, we've also reverse engineered Lawrence Sass' Instant House and prototyped samples in $\boldsymbol{\nabla}$ in order to assess its capabilities and learn from it.

More recently the team also reverse engineered the ClipHut system - from their website pictures and description only - and is beginning to assess its potentials. The most interesting point in the ClipHut model is its use of OSB, while all others use plywood. Also, ClipHut doesn't employ the cassette concept, adopting a rather simpler system akin to traditional wood framing systems and relying entirely on a 'clipping' system for fast assemblage.

\section{Space Allocation and Volume Generation}

Experimentation departed from a preliminary architectural program that would meet the requirements of the Brazilian Social Housing Program - PMCMV , adapting them to the lot and the growth criteria. However, the resulting minimum footage is $54 \mathrm{~m} 2$ of constructed area, higher than the minimum proposed by PMCMV.

Once the characteristics of the architecture and growth orientation were defined, a maximum number of possible dwellers was established, considering the lot size, the growth of the architecture along a single axis and the maximum number of four beds per room. Given the size of the lots and the option for single-family houses, the maximum number of residents was limited to eight, considering that the living room should expand to the limit of a sofa for eight people and the kitchen for a table of up to eight places. Although the minimum room accommodates up to two people, it might receive only one person, impacting the sizing of the other rooms. Thus, supposing a house of three bedrooms to eight people, one of which would house a single person, the others will have to house three and four people. The dialogue with future residents is based on an algorithm developed in Python language, which will ask questions and, depending on the answers, will offer more options, defining the characteristics of the architecture.

A series of questions are proposed to a hypothetical user, from which numerical data is derived and fed into a Python script. Data is then fed into Grasshopper, where plans and volumes and generated.
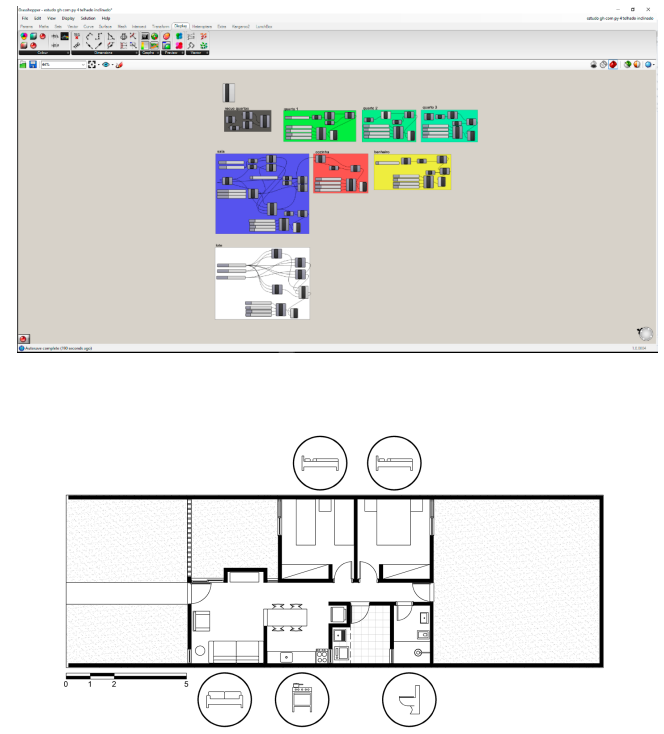

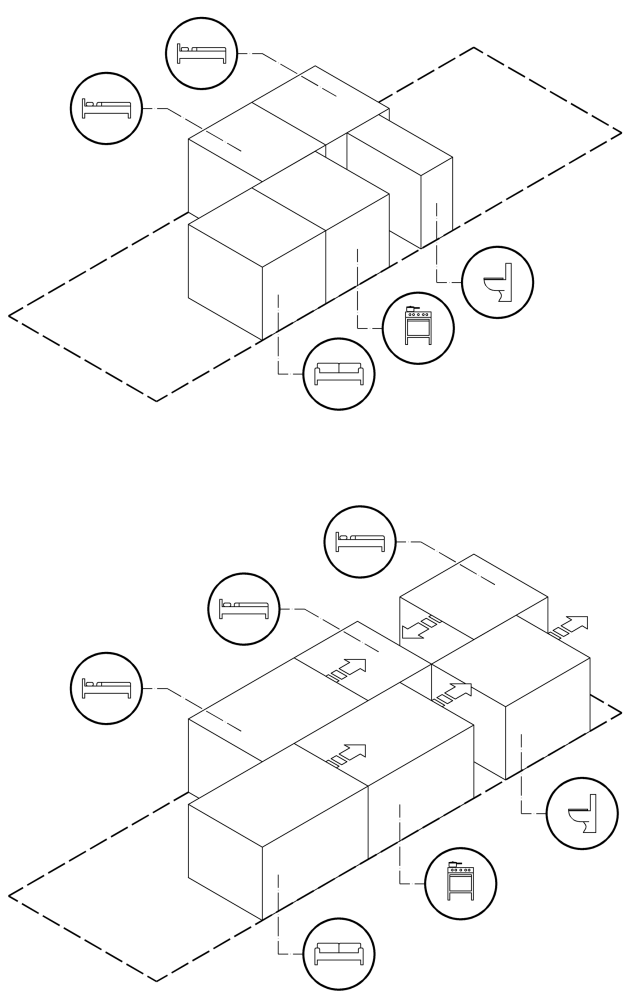

\section{Shop drawings generation}

The connection between the Space Planning module to the Component Design and Fabrication phase might be done in two different ways. Either a series of section lines can be generated directly from Rhinoceros geometry, or the Grasshopper geometry can be fed directly into the Components module. The first one is being used for the ease of operation and because glitches in grasshopper processing large amount of information will not disrupt the work.
Once the transposition from the Massing Model to the porticated structure design model is performed, a series of Grasshopper scripts are employed to parametrically produce shop drawings for CNC machining.

In previous experiments and during the comparison of different wiki systems, testings were carried out to evaluate the performance of all the compared systems and the one being proposed. Machining speed, ease of assembly and structural soundness were accessed.In special, structural soundness for the system behavior using $9 \mathrm{~mm}$ OSB was a key factor, as related in a Paper in SIGraDi 2018.

A $1 / 3$ scale model, laser cut from MDF $3 \mathrm{~mm}$ panels was built, for proof of concept.

During the construction, adjustments in the tonge and groove fittings were made and incorporated into the design. Claddings and other fittings such as doors and windows were modeled in $1 / 3$ and assembled into the model.

As a general method, many adjustments were first designed in Autocad 3d and 2d, and latter coded into the Grasshopper script. This allowed the team to cope rapidly with assemblage problems and to keep the construction pace. Ideally, the scripts should have been edited in the first place, but the team found that disruption in the assemblage was the main issue to be avoided.

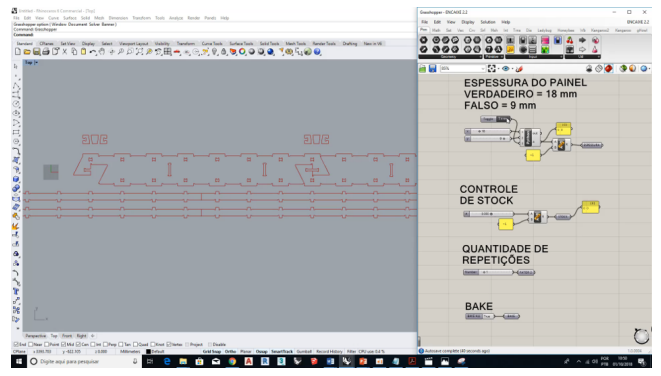

Scripting validation was thus attained by the superimposition of the Grasshopper generated designs onto the Autocad drawings.
Figure 3

Rhinoceros Screen Capture: Volume generation from a Plan variation for 4 occupants.

Figure 4

Rhinoceros Screen Capture: Volume for an eight occupants dwelling.

Figure 5

Grasshopper Screen Capture: scripted generation of the portico segments for fabrication. Variation in stock, machining tolerances, length of segments are set according to modular controls. 
Figure 6

Exploded view of the complete system

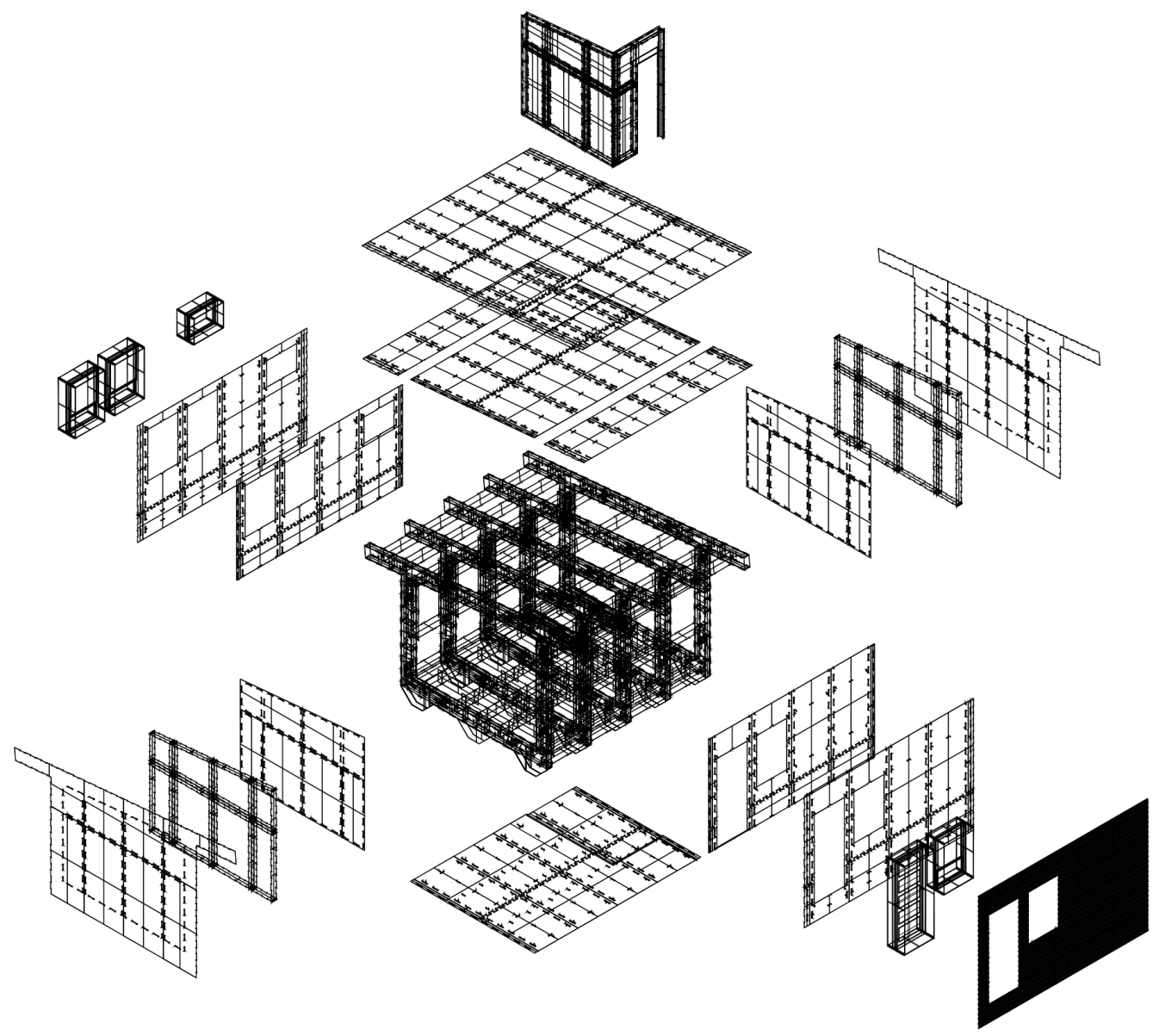




\section{RESULTS}

\section{Plan and Massing generation}

Digital Housing programming tasks have reached the following stages so far:

1. Modularity Control User input dependent Upon selection of boards dimensions - both for the structural system and the cladding systems -, a constraint system limits maximum and minimum spacings. Modularity Control feeds both the 2. Plan Generation module and the 3. Volume Generation Module. Bidirectional control is not possible in the system (which is merely a scripting system in Grasshopper, not allowing for iterations).

2. Plan Generation - User input dependent, meaning that presently we have not reached full automation in space allocation and arrangement. The system is under continuous development and will ultimately be presented with a web interface, allowing users to access main parameters to guide plan generation and choice of materials.

3. Volume Generation: User input dependent after 2. Plan Generation.

4. Parts generation: Automated. All parts are derived directly from the 3 . Volume Generation module and constrained by the 1 . Modularity Control module

\section{Components generation and fabrication}

From the Mass Model in phase 1, parallel section lines spaced accordingly to the construction module are subdivided in Grasshopper and used as a basis for the parametrically generated component design.

Preliminary prototypes in $\otimes$ scale have mostly been generated through the programmed modules, but the modules don't communicate seamlessly yet. Adjustments in the flow of information from one module to the next were studied in this phase, resulting in simpler and faster scripts.

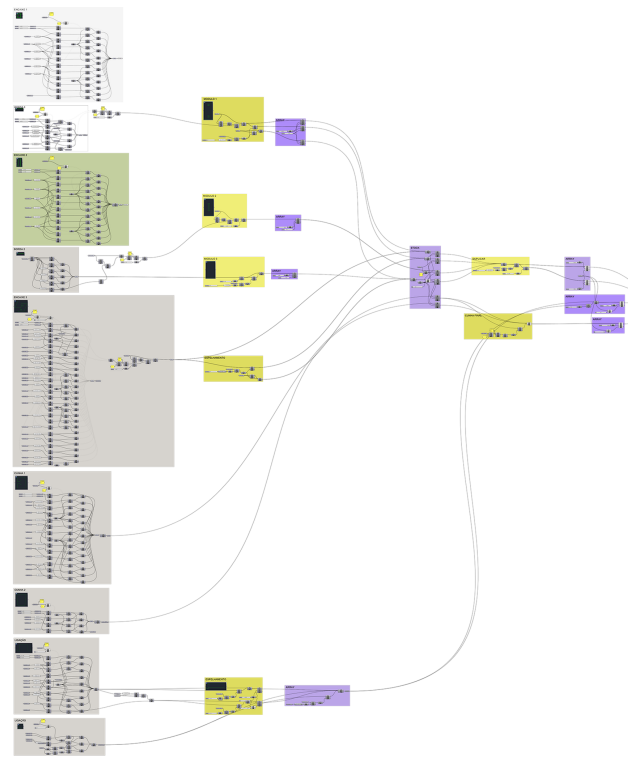

The main prototype before the $1 / 1$ scale model was the $1 / 3$ model, entirely laser cut. This is regarded as our final proof of concept since the majority of the adjustments in the joint systems are tested and adjusted in this scale. Minor adjustments are expected to happen in 1/1 scale. By then, all adjustment systems will be scripted in Grasshopper, allowing for a fast-paced production rate.

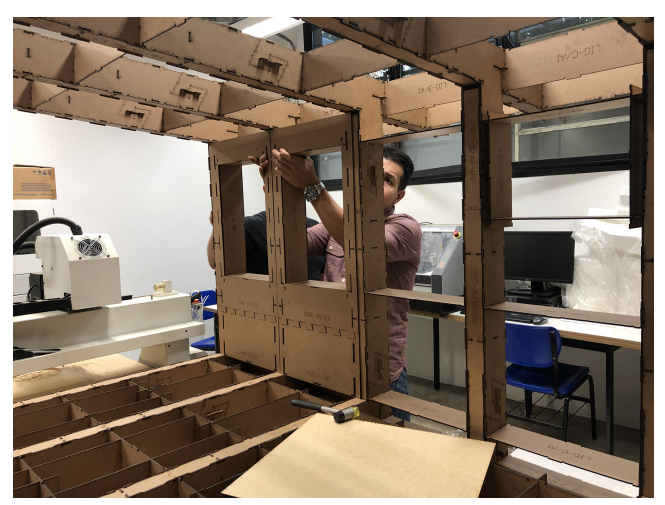

Figure 7

Complete code for the system -

Grasshopper screen capture

Figure 8

$1 / 3$ Scale Prototype cut in 3mm MDF boards, Assembly 


\section{Structural behaviour tests}

A series of structural essays were performed in order to access whether $9 \mathrm{~mm}$ OSB panels would be an acceptable choice for construction, thus ensuring that one of the main goals in this research was met: lower material consumption and lower overall construction cost.

Overall results show that $9 \mathrm{~mm}$ OSB is an entirely acceptable solution.

\section{DISCUSSION \\ Plan and Massing generation}

This research has not gone deeper regarding space layout generation. Presently the code 'embeds' alternatives for layout generation, this meaning that all possible options are 'precoded' into the Grasshopper script.

As such, any new space layout or formal solution should be added by new lines of code. Further exploring space layout generation is not, at the present moment, considered to be a viable solution in this research.

\section{Fabrication System}

Former balloon-frame and the current platformframe construction systems are in the origins of most modern CNC machined systems. Possibly the single most important argued difference is that in Wiki-like systems the whole of construction logic comes embedded in the components, while light framing systems need insitu, qualified workers. However, for a complete working model, many 'non-programmed' operations are still needed to complete a housing project.

Dry construction strategies have taken a long time to occupy significant niches in developing countries such as Brazil, mainly because they rely on industrial plants for mass production of components, but also because mass walls and post and beam concrete systems have dominated both the construction market and, as such, the whole architectural education scene. The architecture education culture thus evolved on the basis of 'open systems', i.e. construc- tion systems not constrained by modularity or prefabricated systems.

The dominance of in Situ concrete pouring into handmade formwork and CMU/plaster have precluded obvious advances such as modular coordination, prefabricated and componentized construction to very small niches in Brazil.

Considering sustainability issues, while the adoption of OSB seems to be a good economic choice, since its raw prices are less than $50 \%$ that of plywood, it should be noted that it poses a problem since "when contrasting OSB with plywood on the basis of sustainability, plywood is a more sustainable structural panel product between the two. [...] production of plywood utilizes lower energy and generates fewer air pollutant emissions of $\mathrm{CO} 2$, SOx, NOx, VOC, and particulate matter." (Chan, 2012)

A study on 'wiki' systems responds to both the immediate need to accelerate housing construction where there's a lack of skilled labor and to gain territory in the architectural education realm, as this project is being done in the University research facilities.

However, the idea of 'programming' construction logic into a 'wiki' system goes in the opposite direction of really empowering local labor with more sophisticated skills. This has been a general trend in the Digital Era: knowledge is embedded in programs and/or programmed objects. From a rather shallow viewpoint, this appears to be to the advantage of poorer populations. However, these populations might as well continue to be set apart from knowledge production and thus not empowered at all.

In developing countries, other difficulties arise. Transportation of produce through long distances in ill-paved roads adds to the costs. Local machining depends on power supply, which is not always available in remote regions. Considering the phasing suggested in Wikihouse.cc, the fourth one - Digital Decentralised seems just so far from reality when developing countries are considered. 


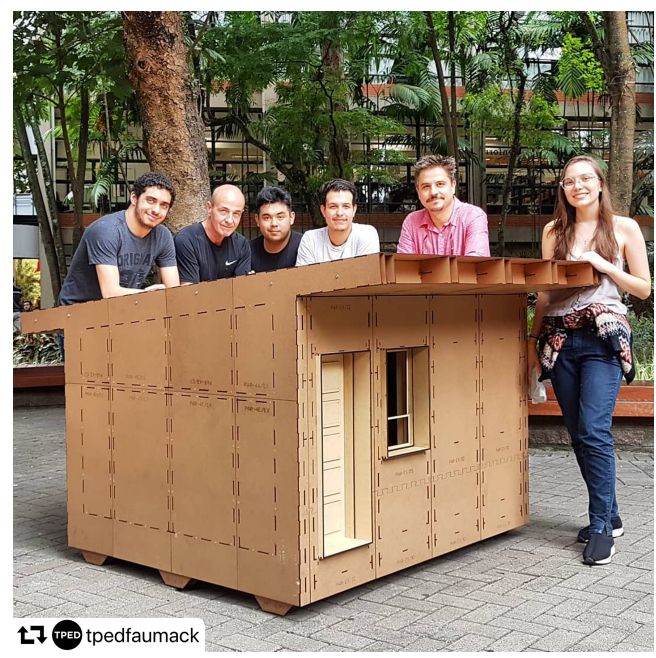

\section{Future development}

The team is beginning the production and construction of the first $1 / 1$ prototype. Final results are expected for 2019, October.

\section{REFERENCES}

Bax, MFTh 1995 'From ideology to methodology: the theoretical evolution of the design methods group', Design research in the Netherlands: a symposium convened by Design Methods Group Information Technology for Architecture, Eindhoven

Campolongo, E and Vincent, CC 2018 'Optimization of a constructive system of subtractive digital fabrication: Prototypes and tests os fitting system', SIGraDi 2018 [Proceedings of the 22nd Conference of the Iberoamerican Society of Digital Graphics - ISSN: 2318-6968] Brazil, São Carlos 7 - 9 November 2018, São Carlos, Brazil, pp. 423-433

Gooren, EMAJ 2017, Shape grammars as a method of design: study on the translation of the architectural design process into the design of a shape grammar, Master's Thesis, Eindhoven University of Technology

Sass, L and Botha, M 2006, 'The Instant House: A Model of Design Production with Digital Fabrication', International Journal of Architectural Computing, 4, pp. 109123
[1] http://www.architectureweek.com/2007/0425/tools 1-1.html

[2] https://www.cliphut.org/
Figure 9

1/3 Scale Prototype complete assembly. 